Supporting Information for

\title{
Zone-Folded Longitudinal Acoustic Phonons Driving Self-Trapped States Emission in Colloidal CdSe Nanoplatelet Superlattices
}

Xinyu Sui, Xiaoqing Gao, Xianxin Wu, Chun Li, Xuekang Yang, Wenna Du, Zhengping Ding, Shengye Jin, Kaifeng Wu, Tze Chien Sum, Peng Gao, Junjie Liu, Xiaoding Wei, Jun Zhang, Qing Zhang, Zhiyong Tang, Xinfeng Liu

*Corresponding: liuxf@nanoctr.cn (X.L.); zytang@nanoctr.cn (Z. T.)

\section{This PDF file includes:}

Experimental Methods

Supporting Note S1: Saturation absorption model for NPLs based on Poisson distribution

Supporting Note S2: Fitting for dynamics with multi-exponential decay function

Supporting Note S3: Coherent oscillations in TA spectroscopy and its signal processing

Supporting Note S4: Wigner-Ville distribution of a time-domain oscillation signal

Supporting Note S5: Calculation for ZFLAP dispersion

Supporting Note S6: Relationship between displacement $\Delta Q$ and Huang-Rhys parameter $S$

Supporting Note S7: Huang-Rhys parameter of CdSe NPL SLs and monodispersed CdSe NPLs

Supporting Note S8: Elastic strain energy distribution in hybrid SL and all-inorganic SL

Supporting Note S9: Relationship between radiative lifetime and Huang-Rhys parameter $S$

Supporting Note S10: The STS emission line shape calculation based on Huang-Rhys parameter $S$

Figure S1. Thickness of CdSe NPL by HAADF-STEM measurement

Figure S2. Center-to-center distance by TEM image analysis

Figure S3. Schematic diagram for stacking configuration of CdSe NPL SLs

Figure S4. Double-Gaussian fitting of PL spectrum of CdSe NPL SLs

Figure S5. TEM images of colloidal CdSe NPL SLs during the sonication-assisted unbundling

Figure S6. UV-VIS absorption spectra of CdSe NPLs during the unbundling process

Figure S7. Continuous evolution of PL spectra during the unbundling process

Figure S8. Power dependent PL spectra and PL excitation spectra of CdSe NPL SLs

Figure S9. XPS for CdSe NPLs

Figure S10. Below bandgap excitation of CdSe NPL SLs in TA spectra

Figure S11. EPR measurement for monodispersed CdSe NPLs

Figure S12. Recovery dynamics for monodispersed CdSe NPLs and NPL SLs

Figure S13. PL lifetime of exciton and STS

Figure S14. Measurement of the pump noise spectrum

Figure S15. Coherent oscillations modulated on STS and exciton in TA spectra of CdSe NPL SLs

Figure S16. Wigner-Ville distribution of coherent oscillations in Figure $3 \mathrm{~b}$

Figure S17.Coherent oscillations modulated on exciton of monodispersed CdSe NPLs

Figure S18. Raman spectroscopy for monodispersed CdSe NPLs and CdSe NPL SLs

Figure S19. Schematic diagram of elastic strain energy distribution

Figure S20. Calculated STS emission line shape with $S=19.9$

Table S1. Gaussian fitting results of PL spectrum of CdSe NPL SLs

Table S2. Analytic peak information of XPS data

Table S3. $g$-factor for electron and hole in $\mathrm{CdX}(\mathrm{X}=\mathrm{S}, \mathrm{Se}, \mathrm{Te})$ nanocrystals

Table S4. Fitting results for the recovery dynamics in TA measurements

Table S5. Multi-Lorentz peak fitting of Figure S18

Table S6. Multi-Lorentz peak fitting of Figure 3d in the main text 


\section{Experimental Methods}

\section{CdSe NPLs synthesis}

Wurtzite CdSe NPLs were synthesized by the reaction of an Se powder and $\mathrm{Cd}(\mathrm{OAm}, \mathrm{OLAm})_{2}$ complex in the mixture solution of octylamine (OAm) and oleylamine (OLAm). To achieve Se powder dispersion, $4.5 \mathrm{mmol}(0.355 \mathrm{~g})$ of Se was mixed with $2.5 \mathrm{~mL}$ OAm and $2.5 \mathrm{~mL}$ OLAm at room temperature with vigorous stirring. To obtain a $\mathrm{Cd}(\mathrm{OAm}, \mathrm{OLAm})_{2}$ complex, $5 \mathrm{~mL}$ of OAm and $5 \mathrm{~mL}$ of OLAm containing $1.5 \mathrm{mmol}(0.275 \mathrm{~g})$ of $\mathrm{CdCl}_{2} \cdot 2 \mathrm{H}_{2} \mathrm{O}$ were heated to $120^{\circ} \mathrm{C}$ for $2 \mathrm{~h}$ and then cooled to room temperature. Se powder dispersion was then injected into an as-prepared $\mathrm{Cd}(\mathrm{OAm}, \mathrm{OLAm})_{2}$ complex solution at room temperature. The final reaction mixture was moderately heated to $100^{\circ} \mathrm{C}$ with a $2^{\circ} \mathrm{C} / \mathrm{min}$ heating rate and then maintained at $100^{\circ} \mathrm{C}$ for $16 \mathrm{~h}$. During this period, the initial solution color changed from black to turbid yellow. After a reaction, excess ethanol containing trioctylphosphine was added, and the sample was precipitated and washed. Finally, the precipitated powder was dispersed in toluene or evaporated to dry powder.

\section{Unbundling Procedure of CdSe NPL}

An aliquot of wurtzite CdSe NPLs saturated solution $(200 \mu \mathrm{L})$ was diluted 15 -fold by toluene to afford a clear yellow dispersion. Then, a solution $(1 \mathrm{~mL})$ of OLAm in toluene was added to the asprepared dilution $(3 \mathrm{~mL})$ to achieve the total concentration of $1 \% \mathrm{v} / \mathrm{v}$ in toluene. The resulting wurtzite CdSe NPLs solution was sonicated vigorously in a bench-top ultrasonic clean machine at room temperature for $6 h$.

\section{Transmission electron microscopy (TEM)}


Samples were dropped in TEM grid on holey-carbon film with the hold of three-layer filter paper. High-resolution TEM image in Figure S1 was achieved with high-angle annular dark-field scanning transmission (HAADF-STEM) mode using Titan Cubed Themis G2 300 (double spherical aberration correction). TEM images in the main text, Figure S2 and Figure S5 were achieved using Tecnai G2 20 S-TWIN at $200 \mathrm{kV}$.

\section{$\underline{\text { Small angle } X \text {-ray scattering (SAXS) }}$}

Small-angle X-ray scattering measurements were carried out on a Xeuss 2.0 instrument equipped with a $\mathrm{Cu}$ X-ray source $(1.5418 \AA)$. Scattering data was collected for $900 \mathrm{~s}$ using collimating slits of $0.8 \times 0.8 \mathrm{~mm}$ ("high flux" mode). Scattering patterns were recorded on a Pilatus $300 \mathrm{~K}$ detector with a sample-to-detector distance of $435 \mathrm{~mm}$ calibrated using a silver behenate standard to achieve a q-range of $0.03-1.0 \AA^{-1}$. Data reduction was performed using the instrument-specific Foxtrot software. Acquiring the distance $(\Delta q)$ between the first and second scattering peak, the NPLs center-to-center distance $d_{0}$ is calculated according to $d_{0}=2 \pi / \Delta \mathrm{q}$.

\section{$\underline{\text { Atomic force microscope (AFM) }}$}

AFM images were obtained by using Bruker Multi Mode 8 AFM, and the scan mode was set at SCANASYST-AIR with Scan Asyst-Air Silicon Nitride probe (Bruker).

\section{Steady state optical spectroscopy}

All spectroscopic measurements for CdSe NPLs were taken with solution conditions. An $800 \mu \mathrm{L}$ portion of each sample was used in a $1 \mathrm{~mm}$ path quartz cuvette. Steady state UV-VIS absorption measurements were taken by a Hitachi U-3010 UV-VIS spectrometer with $\sim 4 \mathrm{~nm}$ bandwidth and 
at the scan speed of $\sim 300 \mathrm{~nm} / \mathrm{min}$. A baseline correction was applied to account for the cuvette and OLAm absorption.

\section{The photoluminescence (PL) and PL excitation (PLE)}

Spectra were obtained at room temperature using a Nano Log spectro-fluorometer (HORIBA Jobin Yvon). In PL mode, excitation wavelength was set at $350 \mathrm{~nm}$ with $2 \mathrm{~nm}$ band-pass filter. The emission light filtered by a $400 \mathrm{~nm}$ long pass filter was collected by a 500/mm blaze grating with 2 $\mathrm{nm}$ bandwidth and at the scan speed of $200 \mathrm{~nm} / \mathrm{min}$. A baseline correction was applied to avoid the OLAm and solvent emission. In PLE mode, the monitor energy was set at the excitonic and selftrapped state peak of $456 \mathrm{~nm}$ and $516 \mathrm{~nm}$, respectively, with the same slit of $5 \mathrm{~nm}$.

\section{Transient absorption spectroscopy}

The femto-second TA measurements were obtained by the HELIOS commercial fs-TA system (Ultrafast Systems). Fundamental $800 \mathrm{~nm}$ pluses (1 kHz, $80 \mathrm{fs})$ from a Coherent Astrella regenerative amplifier were used to pump an optical parametric amplifier (Coherent, OperA Solo) to obtain the frequency-tunable pump beam across the visible region. The pump beam was severed at $500 \mathrm{~Hz}$ and focused at the sample with a beam waist of approximately $300 \mu \mathrm{m}$. A white light continuum probe beam from 430 to $775 \mathrm{~nm}(1.6 \mathrm{eV}$ to $2.9 \mathrm{eV})$ was acquired (with a beam waist of $150 \mu \mathrm{m}$ at the sample) by focusing a small part of the fundamental $800 \mathrm{~nm}$ beam on a sapphire window. The magic angle was set for polarization of the pump and probe respectively to avoid the anisotropic effect. All sample in measurements were in solution and obtained with $1 \mathrm{~mm}$ quartz cuvettes with stirring. Finally, considering the instrument response function of this system, the system had an ultimate temporal resolution of approximately $120 \mathrm{fs}$.

\section{Time-resolved PL spectroscopy}


Time-resolved PL spectroscopy was taken with a NanoLog spectrofluorometer (HORIBA Jobin Yvon) by time-correlated single photon counting mode. A $370 \mathrm{~nm}$ sub-nanosecond $1 \mathrm{MHz}$ pulsed laser diode with $0.1 \mathrm{pJ}$ per pulse (after neutral density filter with $1.0 \mathrm{OD}$ ) was used as the excitation source. The emission light from sample was filtered by a $400 \mathrm{~nm}$ long pass filter and collected by a PMT after a monochromator.

\section{$\underline{\text { Electron paramagnetic resonance (EPR) }}$}

The EPR spectra were measured using the commercial Bruker X/Q-band E580 ELEXSYS spectrometer at X-band (microwave frequency $v$ of $9438.2 \mathrm{MHz}$ ) within the liquid nitrogen temperature $(77 \mathrm{~K})$ to acquire a high signal to noise ratio. The sample was illuminated by a 360 nm CW laser (Changchun New Industries Optoelectronics Tech. Co., Ltd). By reading EPR line in NPL SLs located at magnitude $B_{0}$ of $336.8 \mathrm{mT}$, $g$-factor is calculated according to $g_{S L}=h v / \mu_{\mathrm{B}} B_{0}$, where $\mu_{\mathrm{B}}$ is Bohr magneton.

\section{Low-frequency Raman spectroscopy}

All the Raman spectra were excited by a $532 \mathrm{~nm} \mathrm{CW}$ laser and collected using a confocal triplegrating $(1800 \mathrm{~g} / \mathrm{mm})$ spectrometer in subtract low-wave number mode (Horiba-JY T64000). Pump and collection beam were polarized in parallel with the help of a pair of polaroid films (Thorlabs, LPVISE100-A). 


\section{Supporting Notes}

Supporting NoteS1: Saturation absorption model for NPLs based on Poisson distribution

The probability of the photon absorption by a NPL as a function of photon number $n$ is considered as the Poisson distribution: $P(<n>)=<n>^{n} \mathrm{e}^{-<n>} / n$ !, where $<n>$ represents the average photon number absorbed by each NPL. $<n>$ can be associated with pump fluence $I$ with a scaling factor C in the form of $\langle n\rangle=C I$. Absolute TA signal $|\Delta A(I)|$ as a function of pump fluence $I$ is proportional to the probability of all absorbed photon counts except the zero condition in the formula:

$$
|\Delta A(I)|=\gamma\left[1-P_{0}(<n>)\right]=\chi\left(1-e^{-<n>}\right)=\chi\left(1-e^{-C L}\right)
$$

In the main text Figure $2 \mathrm{c}$, both bleach maximum of component $\mathrm{X}$ and $\mathrm{Y}$ were normalized from 0 to $1 . \mathrm{C}_{\mathrm{X}}$ was fitted as $0.065 \pm 0.003 \mathrm{~cm}^{2} / \mu \mathrm{J}$ and $C_{Y}$ was fitted as $0.067 \pm 0.003 \mathrm{~cm}^{2} / \mu \mathrm{J} . \gamma_{X}$ was fitted as $0.995 \pm 0.001 ; \gamma_{Y}$ was fitted as $0.995 \pm 0.001$.

Supporting NoteS2: Fitting for dynamics with multi-exponential decay function

The bleach recovery signal was fitted by multi-exponential decay function with life time $\square_{i}$ convoluted by a Gaussian response function:

$$
D(t)=y_{0}+\exp \left(\frac{t}{\tau_{i}}\right)\left[1-\operatorname{erf}\left(-\frac{t-\frac{s_{0}^{2}}{\tau_{i}}}{s_{0}}\right)\right]
$$

where $i$ is set as $3, \mathrm{y}_{0}$ is baseline height, $\mathrm{s}_{0}$ is laser pulse width. For each $\tau_{i}$, its proportion $p_{i}$ is calculated by $p_{i}=\mathrm{A}_{\mathrm{i}} \tau_{i} / \Sigma \mathrm{A}_{\mathrm{i}} \tau_{i}$. Fitting results were given in Supporting Table S4.

Supporting NoteS3: Coherent oscillations in TA spectroscopy and its signal processing 
Coherent oscillations superimposed on the recover kinetics as a function of delay time in the TA spectroscopy stem from the modulation of coherent lattice vibrations. With electron-phonon coupling, potential surface of a certain electron state is periodically deviated from the balance position, which gives rise to the modulation of absorption or emission of the electron state. This modulation is directly reflected by the intensity change of probe beam. By subtracting carrier population dynamics from TA profile, the oscillation signal is fully presented in the residual parts. Via fast Fourier transform (FFT), the vibrational spectrum can be extracted and quantitatively analyzed. The detailed signal acquisition and analysis procedure are presented below.

First, fluctuation noise from pump laser should be acquired as a baseline for spectrum analysis. In this work, we measured the TA profile of organic photovoltaic semiconductor such as Poly(3-hexylthiophene) to acquire the laser fluctuations (Figure S14). The residual part contained the laser fluctuations after the subtraction of exponential population decay.

Second, vibrational oscillations from the sample are acquired with population background subtraction (Figure S15). The residual part contained the vibrational signal as well as the laser fluctuations.

Third, we perform the FFT for both pump fluctuations and vibrational oscillations to acquire their frequency spectrum. In order to minimize the spectrum leakage, Kaiser window was used for all time domain signals. The window function can be written as:

$$
\mathrm{w}(n)=I_{0}\left(\beta \sqrt{ }(1-((1-N / 2) / N / 2))^{2}\right) / \mathrm{I}_{0}(\beta), 0 \leq n \leq \mathrm{N},
$$

where $I_{0}$ is the zeroth-order modified Bessel function of the first kind, $\mathrm{N}+1$ represents the total length of the signal $\mathrm{L}(\mathrm{L}=\mathrm{N}+1)$.

Fourth, the noise spectrum should be normalized as the baseline for vibrational spectrum. The pulse width limited frequency resolution is $238 \mathrm{~cm}^{-1}$ according to the convolution width of pump and probe pulses with $\sim 140$ fs. The vibrational and noise spectra are normalized in spectra 
range above $238 \mathrm{~cm}^{-1}$ where both them share the same system noise. Finally, we can distinguish phonon spectra below $238 \mathrm{~cm}^{-1}$.

Supporting Note S4: Wigner-Ville distribution of a time-domain oscillation signal

We perform the time-frequency analysis of the vibrational signal via Wigner-Ville distribution. For a continuous signal $x(t)$ in the time domain, the Wigner-Ville distribution $W V D_{x}(t, f)$ as a function of time $t$ and frequency $f$ is defined as

$$
W V D_{x}(t, f)=\int_{-\infty}^{\infty} x\left(t+\frac{\tau}{2}\right) x^{*}\left(t-\frac{\tau}{2}\right) e^{-i 2 \pi f \tau} d \tau
$$

The Wigner-Ville distribution of signal from Figure $3 \mathrm{~b}$ is shown in Figure S16.

\section{Supporting NoteS5: Calculation for ZFLAP dispersion}

To calculate the phonon dispersion, we use the simplest diatomic linear chain model with the dispersion function below:

$$
\omega_{ \pm}^{2}(q)=\frac{\beta}{M_{1} M_{2}}\left\{\left(M_{1}+M_{2}\right) \pm\left[M_{1}^{2}+M_{2}^{2}+2 M_{1} M_{2} \cos (q a)\right]^{1 / 2}\right\}
$$

where $\mathrm{M}_{1}\left(112.41 m_{\mathrm{a}}\right)$ and $\mathrm{M}_{2}\left(78.96 m_{\mathrm{a}}\right)$ represent the relative mass of $\mathrm{Cd}$ and $\mathrm{Se}$ atom, $\beta=840000 \mathrm{~cm}^{-1} / m_{\mathrm{a}}$, and $m_{\mathrm{a}}$ represents the relative atomic mass. The optic and acoustic modes were separated with positive and negative sign of \pm symbol. For the CdSe NPL SLs in this work, the mini- Brillouin zone was folded for about 7 times discussed in the main text. In addition, considering the intrinsic folded nature in WZ structure, ${ }^{1}$ the ZFLAP was finally presented in Figure $3 \mathrm{e}$ in the main text.

Supporting Note S6: Relationship between displacement $\Delta Q \underline{Q}$ and Huang-Rhys parameter $S$

The self-trapping energy $E_{S T}$ is contributed by ZFLAP participation with value of $E_{S T}=S \Omega_{Z F L A P}$. $E_{S T}$ can be written as $E_{S T}=1 / 2 \Omega_{Z F L A P} \Delta Q^{2}$ under harmonic approximation, where $\Delta Q$ is the 
dimensionless normal coordinate. Combine above two expressions, $\Delta Q$ can be directly linked with Huang-Rhys parameter $S$ with $\Delta Q=\sqrt{ } 2 S$.

Supporting Note S7: Huang-Rhys parameter of CdSe NPL SLs and monodispersed CdSe NPLs

For CdSe NPL SLs, S can be calculated through the following equation: $\sqrt{\mathrm{S}}=\frac{2.36 \hbar \Omega \sqrt{\operatorname{coth} \frac{\hbar \Omega}{2 k_{B} T}}}{F W H M}$, where $k_{B}$ is the Boltzmann constant, $T$ is the temperature, and FWHM is the emission broadening of STS. ${ }^{2}$ Substituting the FWHM as $\sim 228 \mathrm{meV}, \hbar \Omega \sim 9.2 \mathrm{meV}, S$ is inferred to be $\sim 19.9$.

The above calculation for Huang-Rhys parameter is suitable for the phonon dominated emission broadening, which is the condition of strong EPC. ${ }^{3}$ Due to the weak EPC, the emission broadening in monodispersed CdSe NPLs is less influenced by phonons, but more affected by other factors like degree of quantum confinement, the density of surface states. ${ }^{4}$ To evaluate a more precise Huang-Rhys parameter, we took an different approach which uses the relationship between stokes shift of emission and Huang-Rhys factor of $S=\Delta_{\text {stokes }} / 2 \hbar \Omega$, where $\Delta_{\text {stokes }}$ represents the Stokes shift, $\hbar \Omega$ is the energy of the involved phonons. ${ }^{5}$ The Stokes shift of monodispersed CdSe NPLs is $1 \mathrm{~nm}$ (PL at $452 \mathrm{~nm}$ and absorption at $451 \mathrm{~nm}$ ), corresponding to $\Delta_{\text {stokes }}=6 \mathrm{meV}$. Substituting optical phonon energy as approximately $25.5 \mathrm{meV}\left(205.9 \mathrm{~cm}^{-1}\right.$, Table S5), $S$ is calculated as $\sim 0.1$.

Supporting Note S8: Elastic strain energy distribution in hybrid and all-inorganic SL

A mechanical model discussed below show the relative elastic strain energy distributions in the SL structure. As is illustrated in Figure S19, we simplify two types of layer in SL along stacking direction into two types of springs with different stiffness coefficients $k$.

In hybrid organic-inorganic SL of CdSe NPL (Figure S19A), the stiffness coefficients are labeled as $k_{0}$ and $k_{l}$ for the inorganic part and organic part, respectively. As a comparison, we 
introduce all inorganic SL where original organic layer is replaced by inorganic CdTe layer in Figure 19B. The stiffness coefficient for CdTe is labeled as $k_{2}$. It is natural to conclude that stiffness coefficients of CdSe and CdTe are comparable and both them are much larger than the stiffness coefficient of organic layer $\left(k_{0} \approx k_{2}>k_{1}\right)$. For the small displacement $\delta l$ induced by the lattice vibration, the elastic strain energy $V$ for $\mathrm{CdSe}$, organic ligands, and $\mathrm{CdTe}$ is written as $V_{0}=1 / 2 k_{0} \delta l^{2}, V_{1}=1 / 2 k_{1} \delta l^{2}, V_{2}=1 / 2 k_{2} \delta l^{2}$, respectively. With $k_{0} \approx k_{2} \geq k$, it can be deduced that $V_{0} / V_{1}>$ $V_{0} / V_{2}$, which means that the strain energy prefers to concentrate in the inorganic part of hybrid SL compared with uniformly distribution in all inorganic SL system.

Supporting Note S9: Relationship between radiative lifetime and Huang-Rhys parameter $S$

Radiative probability is expected to proportional to Franck-Condon factor $F . F$ is a function of Huang-Rhys parameter $S$, described as $F(\mathrm{~S})=\left|\left\langle\chi_{G S} \mid \chi_{E S}\right\rangle \quad\right|^{2}=e^{-S} S^{S} / S !$, where $\chi_{G S}$ and $\chi_{E S}$ are the vibrational wavefunctions of ground state and excited state, respectively. Hence, the ratio of radiative lifetime (the inverse of radiative probability) of CdSe NPL SLs $\left(\tau_{S L s}\right)$ and monodispersed CdSe NPLs $\left(\tau_{\text {mono }}\right)$ can be written as $\tau_{S L s} /\left.\tau_{\text {mono }}\right|_{\text {calculation }}=F\left(S_{\text {mono }}\right) / F\left(S_{S L s}\right)$ and calculated as 8.5 , according to $S_{\text {mono }}=0.1$ and $S_{S L s}=19.9$. By comparison, the experimental ratio as $\tau_{S L s} / \tau_{m o n o} \mid$ experiment $=3.0 \mathrm{~ns} / 0.36 \mathrm{~ns}=8.3$ is in good agreement with the calculated value (8.5).

Supporting NoteS10: The STS emission line shape calculation based on Huang-Rhys parameter $\underline{S}$

The emission energy of STS could be calculated as $E_{P L}=E_{e}-E_{S T}-E_{d}$, where $E_{\mathrm{e}}$ represents the band edge emission energy, $E_{\mathrm{ST}}$ represents the self-trapping energy and $E_{\mathrm{d}}$ represents the lattice deformation energy. ${ }^{6}$ Because both $E_{\mathrm{ST}}$ and $E_{\mathrm{d}}$ are the energy reduction induced by EPC, it can be 
approximate that $E_{P L}=E_{d} . E_{P L}$ is further simplified and written as $E_{P L}=E_{e}-2 E_{S T}$. In the main text, $E_{\mathrm{ST}}$ is simply expressed as $E_{\mathrm{ST}}=S \hbar \Omega_{p}$, where $\hbar \Omega_{p}$ represents the average phonon energy $(9.2 \mathrm{meV}$ in the main text) and $S$ represents the Huang-Rhys parameter. Considering phonon number $m$ obeys Poisson distribution with expectation of $S, E_{S T}$ can be rewritten as $E_{\mathrm{ST}}(m)=e^{-s}\left(S^{\mathrm{m}} / \mathrm{m} !\right) \hbar \Omega_{p}{ }^{7}$ Then $E_{P L}$ can be written as $E_{P L}(m)=E_{e}-2 E_{S T}(m)=E_{e}-2 \sum_{n=0}^{\infty} E_{S T}(m)$. Based on the expression of $E_{P L}$, emission line shape for STS is calculated in Figure S20. 
A

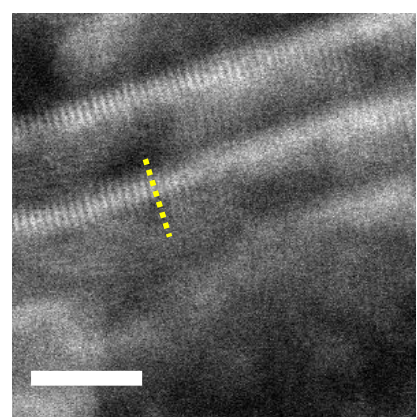

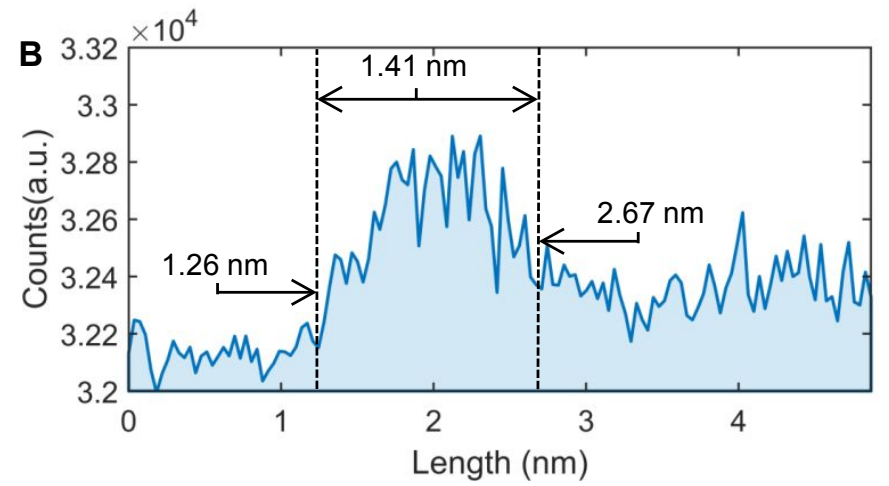

Figure S1 Thickness of CdSe NPL by HAADF-STEM measurement. (A) HAADF-STEM image of a selected section along [11-20] axis. Scale bar is $5 \mathrm{~nm}$. Yellow dashed line represents the interested section analyzed in (B). Thickness of inorganic framework of CdSe NPLs with 1.41 nm analyzed by GATAN Digital Micrograph software. 


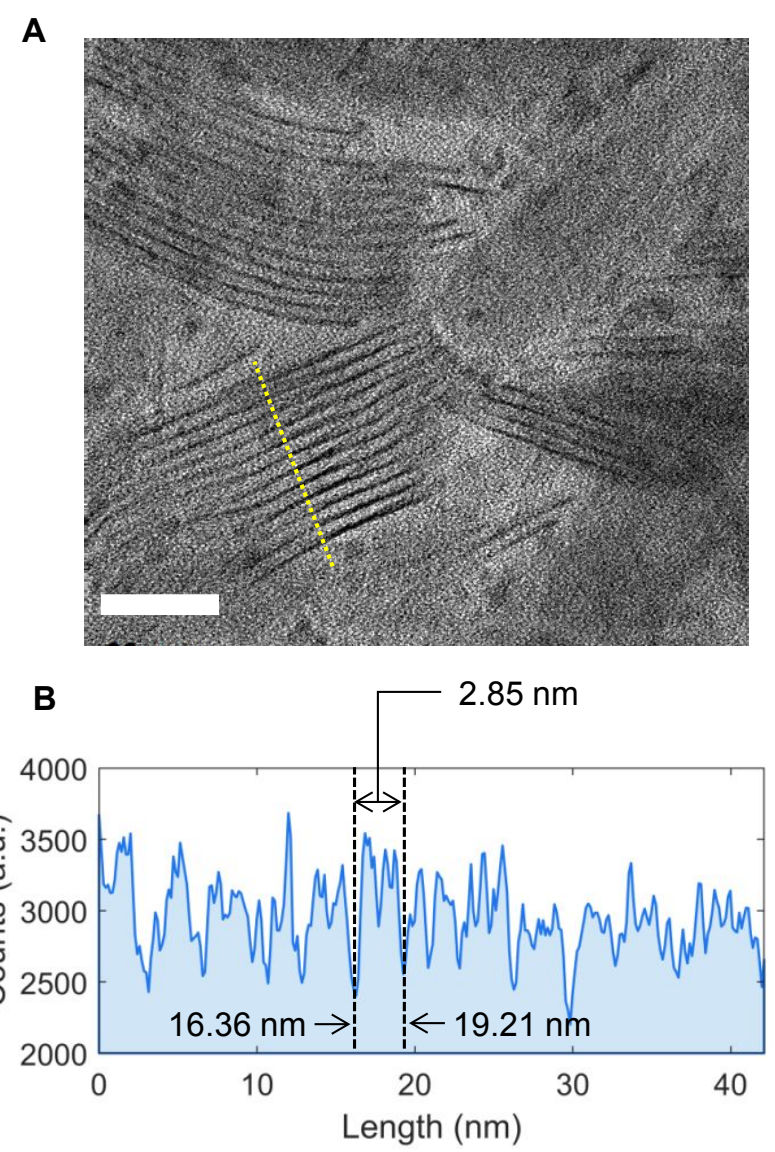

Figure S2 Center-to-center distance by TEM image analysis. (A) TEM image of CdSe NPL SLs. Scar bar is $20 \mathrm{~nm}$. Yellow dashed line represents the interested section for analysis. (B) Center-to-center distance of CdSe NPL SL is $2.85 \mathrm{~nm}$ analyzed by GATAN Digital Micrograph software. 


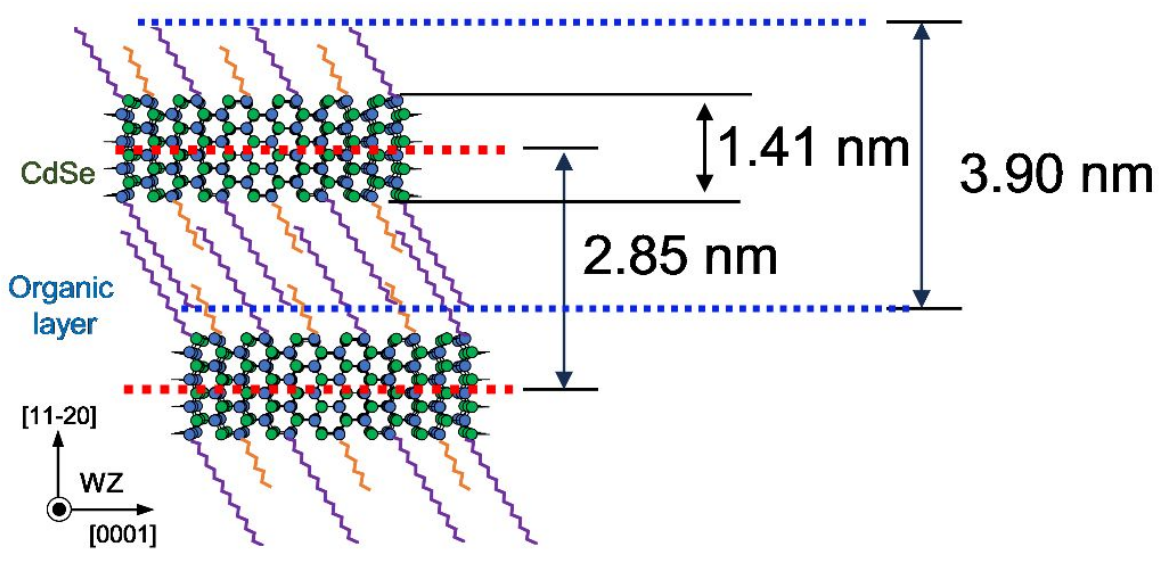

Figure S3 Schematic diagram for stacking configuration of CdSe NPL SLs. Center-to-center distance $(2.85 \mathrm{~nm})$, thickness of the inorganic part of NPL $(1.41 \mathrm{~nm})$ and the whole thickness of CdSe NPL containing ligand (3.90 nm) are labeled, respectively. 


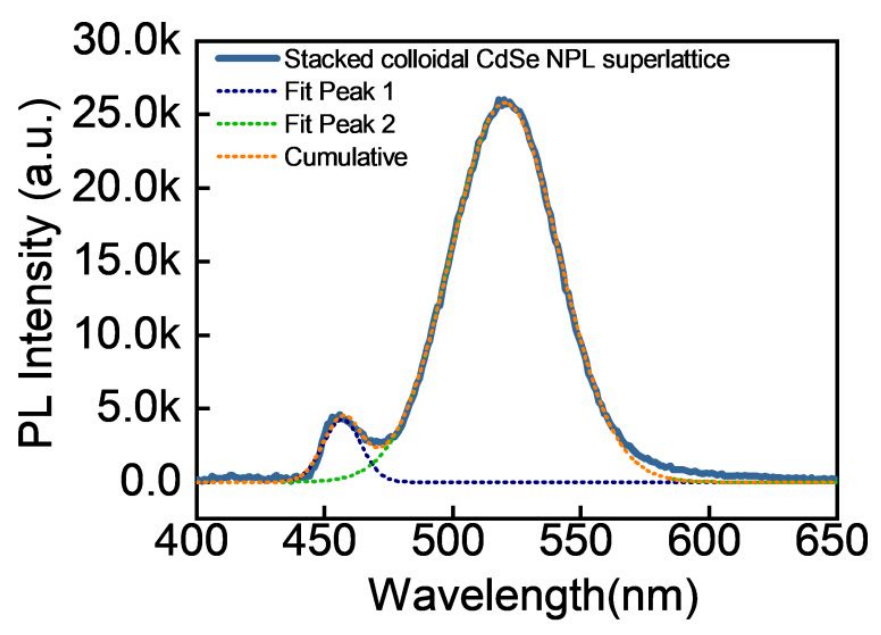

Figure S4 Double-Gaussian fitting of PL spectrum of CdSe NPL SLs. Fitting function is described as $I(E)=\sum_{i} \frac{A_{i}}{w_{i} \sqrt{\frac{\pi}{2}}} e^{-2 \frac{\left(E-P_{i}\right)^{2}}{w_{i}^{2}}}, i=1,2$, where $A_{\mathrm{i}}$ represents the area of fitting peak, $P_{\mathrm{i}}$ represents the fitting peak center and $w_{\mathrm{i}}$ represents the FWHM. The resulting fitting parameters were presented in Table S1. 
A
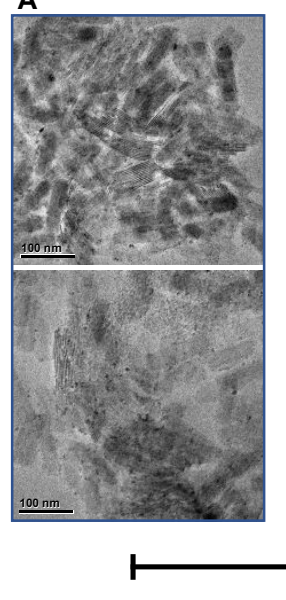

$0 \min$
B

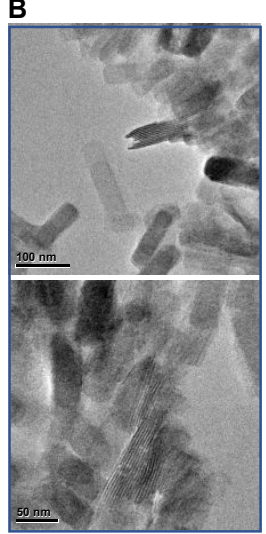

$30 \mathrm{~min}$
C
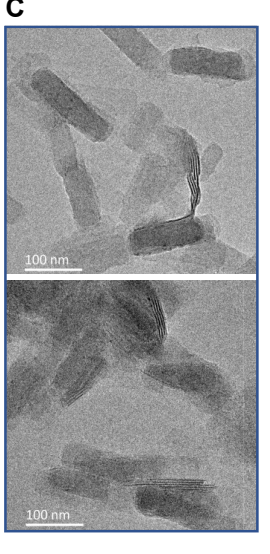

$60 \mathrm{~min}$
D

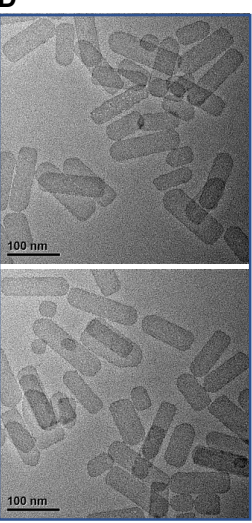

Sonication Time

$300 \min$

Figure S5 TEM images of colloidal CdSe NPL SLs during the sonication-assisted unbundling

process. (A-D) were acquired at 0, 30, 60 and 300 minutes respectively in sonication processes. 


\section{A}

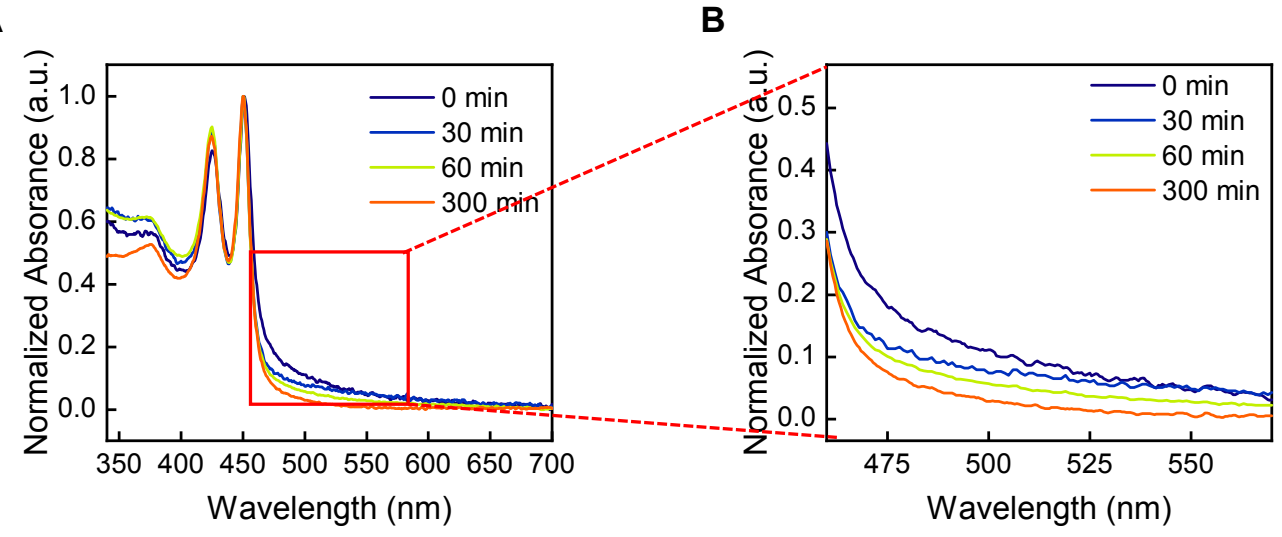

Figure S6 UV-VIS absorption spectra of CdSe NPLs during the unbundling process. (A) All spectra were normalized at first absorption peak for line shape comparison. Zoom in plot (B) shows the decreased scattering induced tail, which is in accord with the result in Ref.8. 


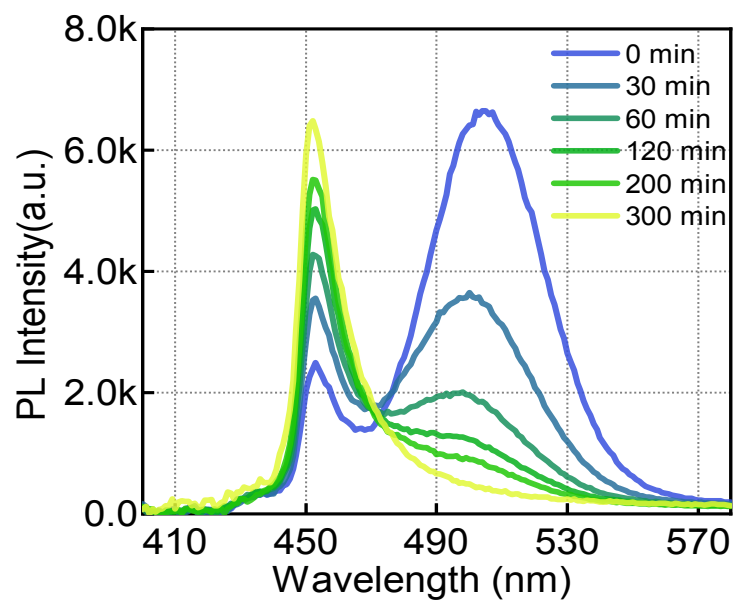

Figure S7 Continuous evolution of PL spectra during the unbundling process. With the sonication-assisted unbundling from 0 to $300 \mathrm{~min}$, the darkening of STS emission and the brightening of excitonic emission are observed. 

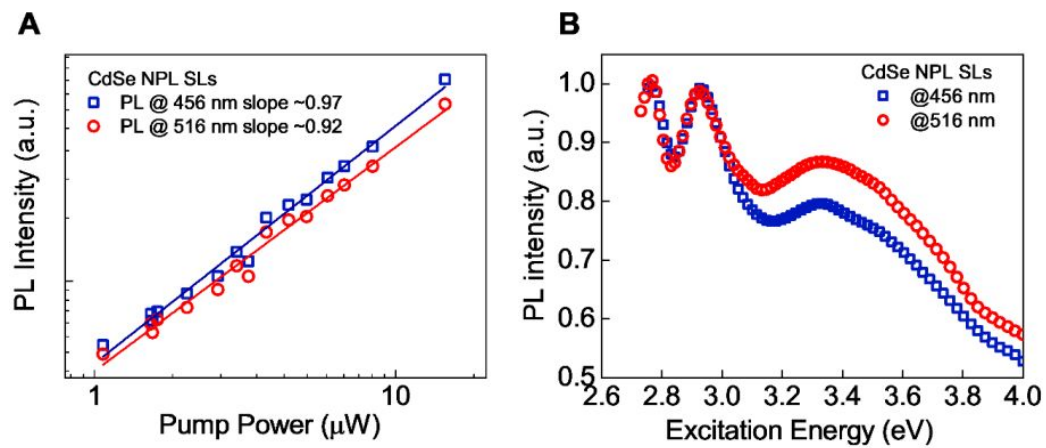

Figure S8 Power dependent PL spectra and PL excitation spectra of CdSe NPL SLs. (A) PL intensity of excitonic emission $(456 \mathrm{~nm})$ and the STS emission $(516 \mathrm{~nm})$ as a function of pump power. (B) PL excitation spectra monitored at the exciton emission peak (456 nm) and STS emission peak $(516 \mathrm{~nm})$, respectively. 

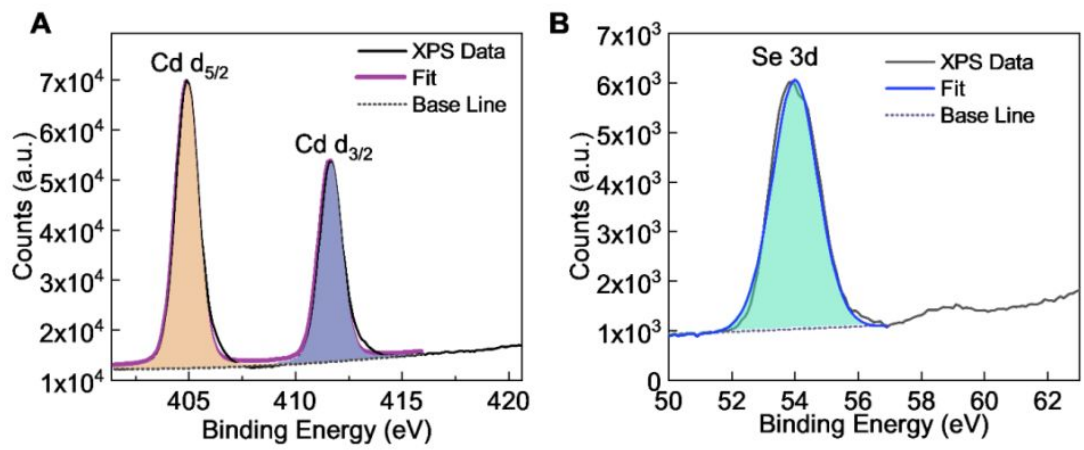

Figure S9 XPS for CdSe NPLs. Close-up surveys for Cd 3d (A) and Se 3d (B) cores. Both spectra were acquired with same scan rates and times in order the quantitative analysis. All peaks were fitted with Voigt function after subtracting Tougaard background (fitting result see Table S2). 


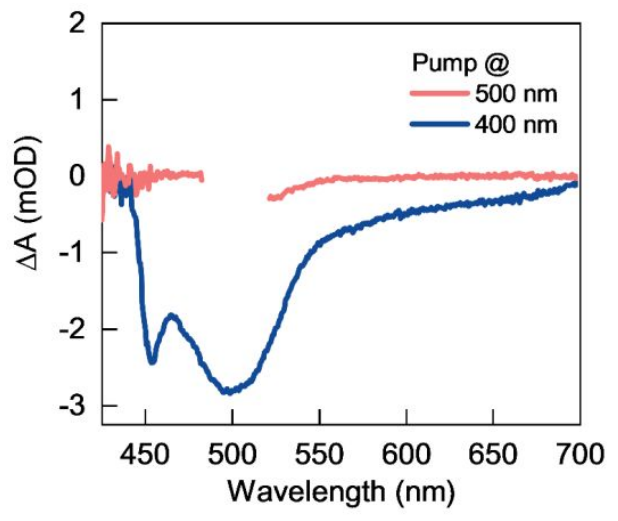

Figure S10 Below bandgap excitation of CdSe NPL SLs in TA spectra. TA spectra pumped with $400 \mathrm{~nm}$ and $500 \mathrm{~nm}$ with fluence of $2.7 \mu \mathrm{J} / \mathrm{cm}^{2}$, respectively. The time delay is fixed at $1 \mathrm{ps}$. 


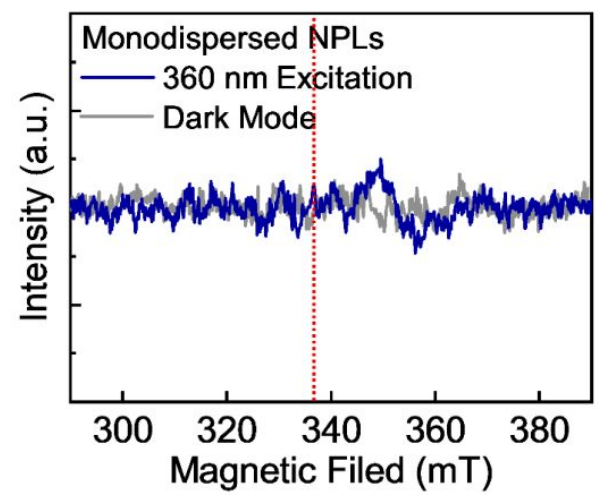

Figure S11 EPR measurement for monodispersed CdSe NPLs. The red dashed line indicates the position of EPR signal for CdSe NPL SLs with $g=2.002$ in the main text. 

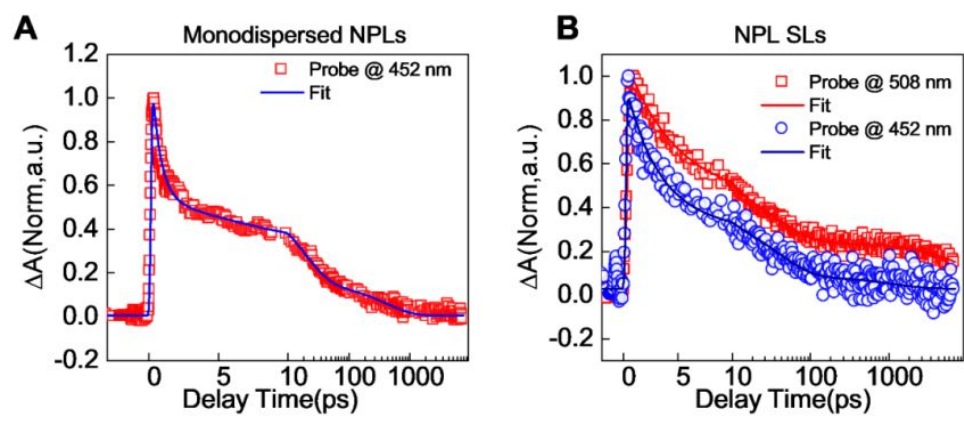

Figure S12 Recovery dynamics for monodispersed CdSe NPLs and NPL SLs. (A) Normalized $\Delta \mathrm{A}$ intensity as a function of delay time probed at $452 \mathrm{~nm}$ (exciton state) for monodispersed CdSe NPLs. (B) Normalized $\Delta \mathrm{A}$ intensity as a function of delay time probed at $452 \mathrm{~nm}$ (exciton state) and $508 \mathrm{~nm}(\mathrm{STS})$, respectively. 
A

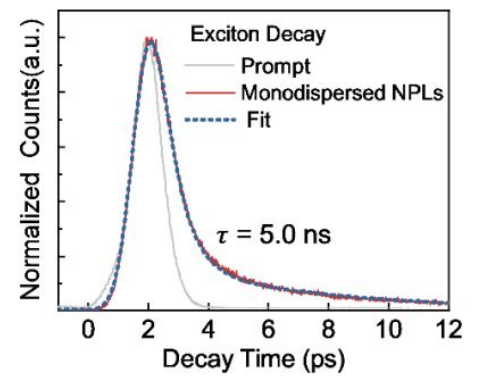

B

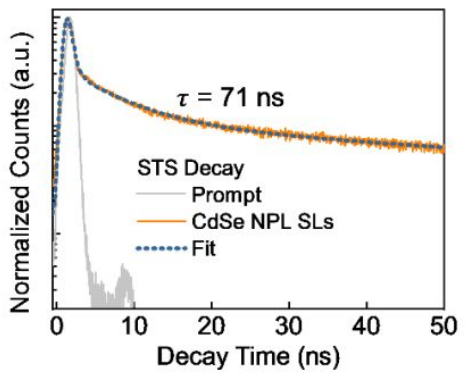

Figure S13 PL lifetime of exciton and STS. (A) The exciton emission lifetime in CdSe monodispersed NPLs is 5.0 ns. (B) The STS emission lifetime in CdSe NPL SLs is 71 ns. IRF in both figures represent the instrument response function. 
A

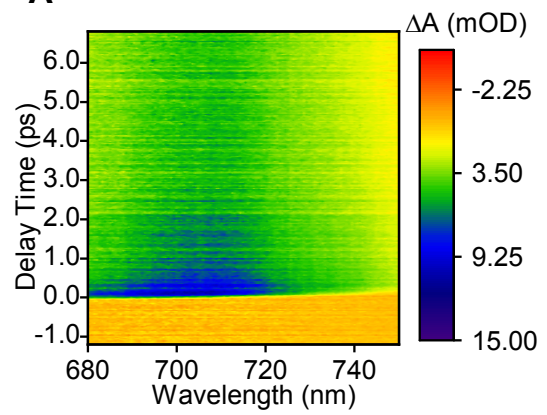

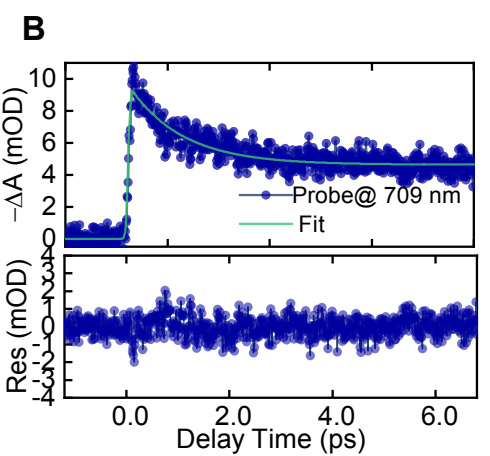

C

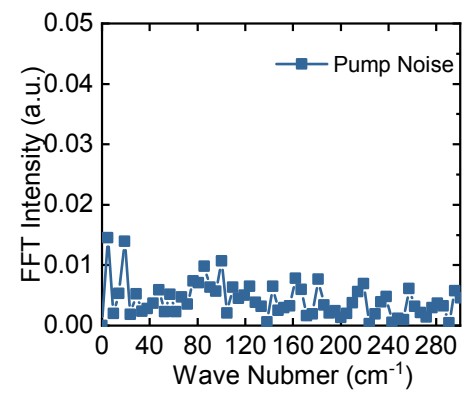

Figure S14 Measurement of the pump noise spectrum. (A) TA spectra of a Poly(3hexylthiophene) film with $400 \mathrm{~nm}$ excitation. (B) The decay dynamics probed at $709 \mathrm{~nm}$ and its exponential decay fitting shown in upper panel. Residual result is shown in down panel. (C) FFT of the residual with Kaiser window. 
A

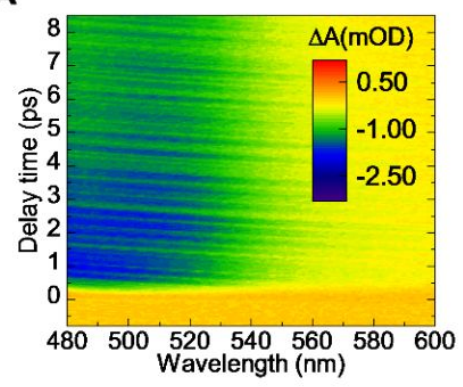

B

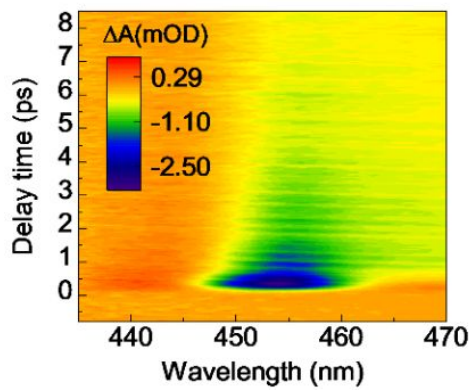

C

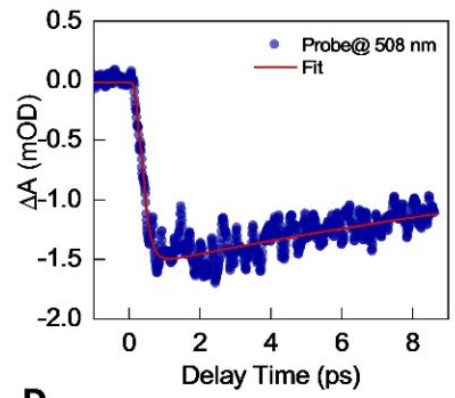

D

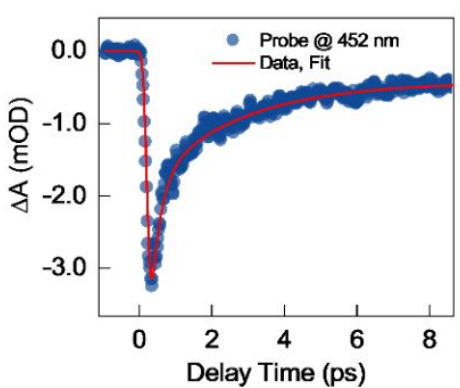

E

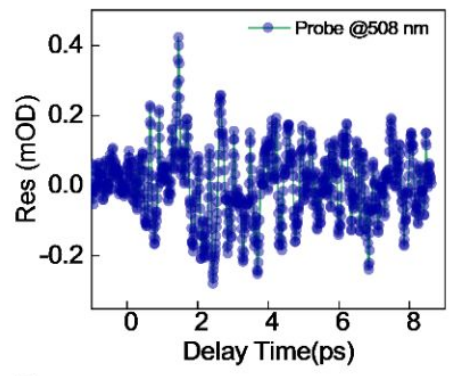

F

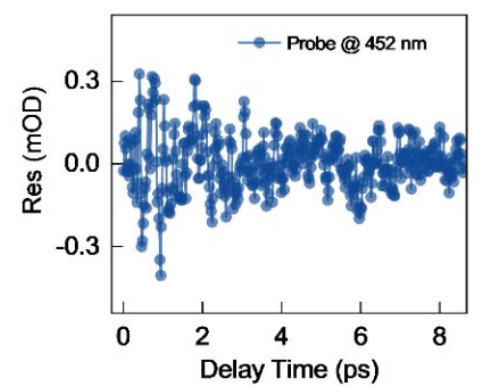

Figure S15 Coherent oscillations modulated on STS and exciton in TA spectra of CdSe NPL

SLs. (A-B) Two-dimensional pseudo-color mapping of TA for CdSe NPL SLs under $400 \mathrm{~nm}$ excitation. (C-D) The population dynamics for STS (probed at $508 \mathrm{~nm}$ ) and exciton (probed at 452 $\mathrm{nm}$ ), respectively. (E-F) The residual parts by subtraction population backgrounds of STS and exciton, respectively. 


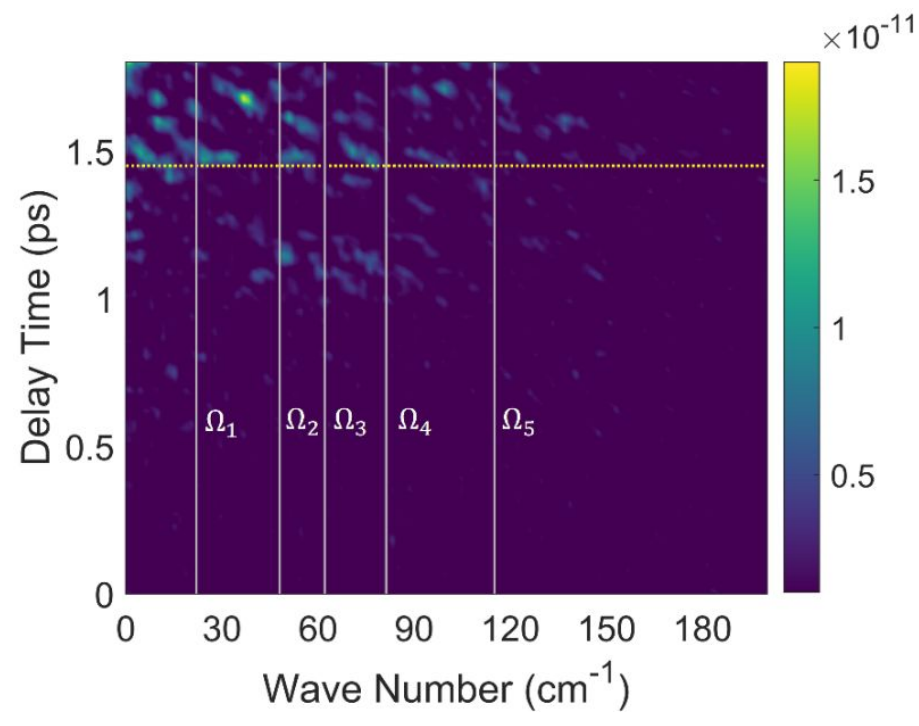

Figure S16 Wigner-Ville distribution of coherent oscillations in Figure $3 \mathrm{~b}$. The frequency evolution as a function of delay time originated from the oscillations modulated on the STS in TA spectra. Yellow dashed line highlights that $\Omega_{1}$ to $\Omega_{5}$ all work together simultaneously. 

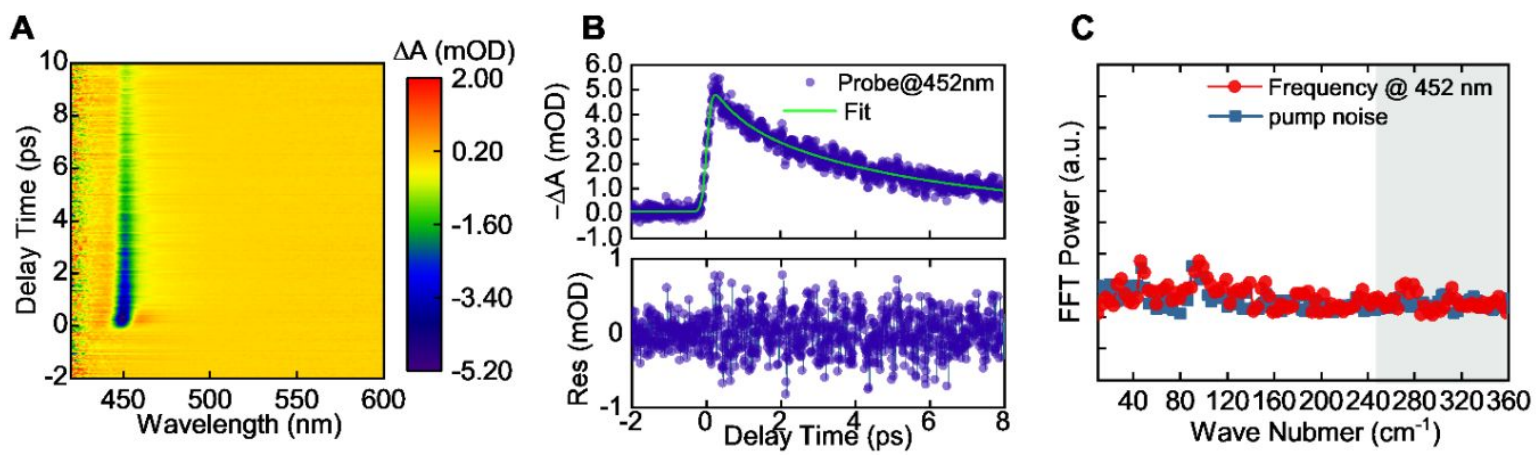

Figure S17 Coherent oscillations exciton of monodispersed CdSe NPLs. (A) TA spectra of monodispersed colloidal CdSe NPLs with $400 \mathrm{~nm}$ excitation. (B) The exciton decay dynamics (probed at $452 \mathrm{~nm}$ ) and its exponential fitting (upper panel). Residual result was shown in down panel. FFT of the residual signal with Kaiser window was shown in (C). In addition, the pump noise (blue line) was normalized in the frequency band labeled in grey for baseline. 


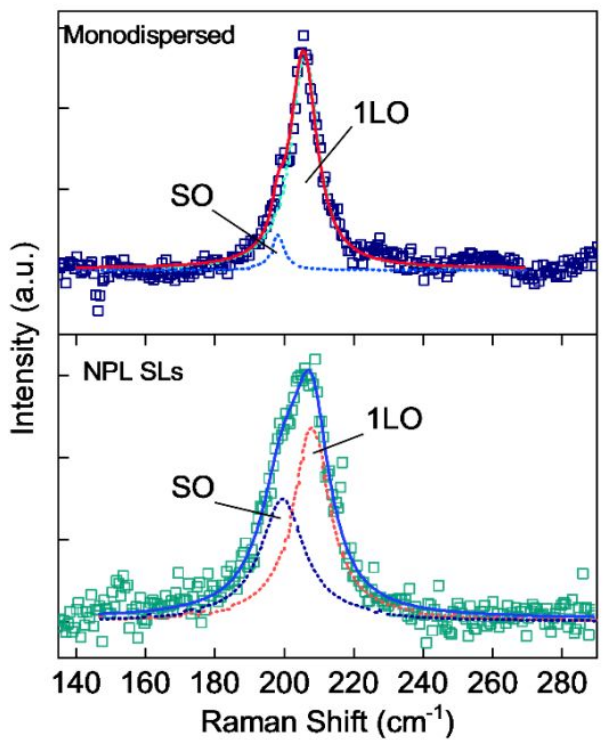

Figure S18 Raman spectroscopy for monodispersed CdSe NPLs and CdSe NPL SLs. The first optical phonon mode (1LO) and surface optical phonon (SO) are labeled respectively. MultiLorentz peak fitting results is summarized in Table S5. 

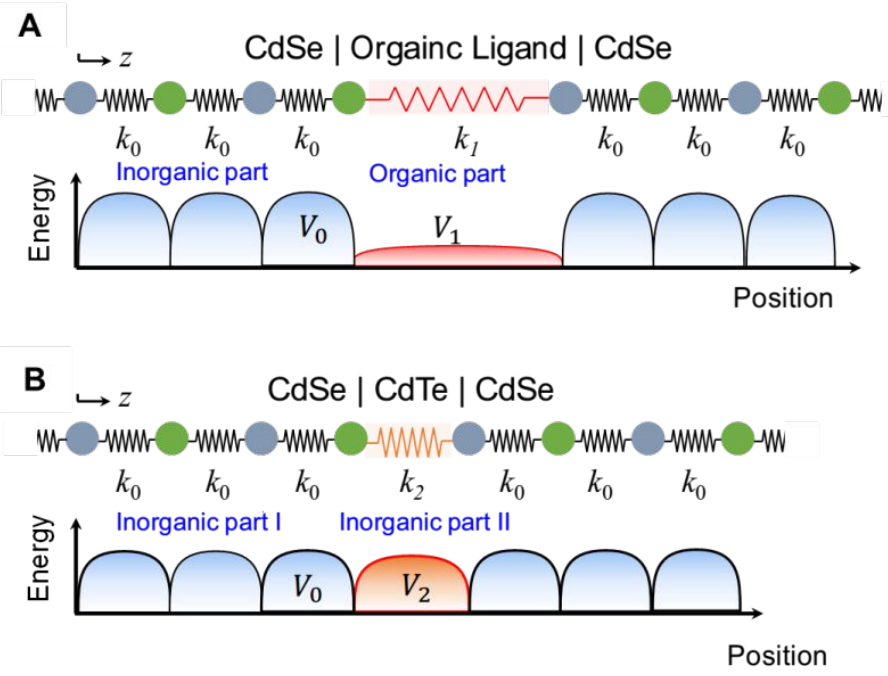

Figure S19 Schematic diagram of elastic strain energy distribution. Elastic strain energy distributions along the stacking directions in (A) hybrid organic-inorganic SLs, and (B) allinorganic SLs. 


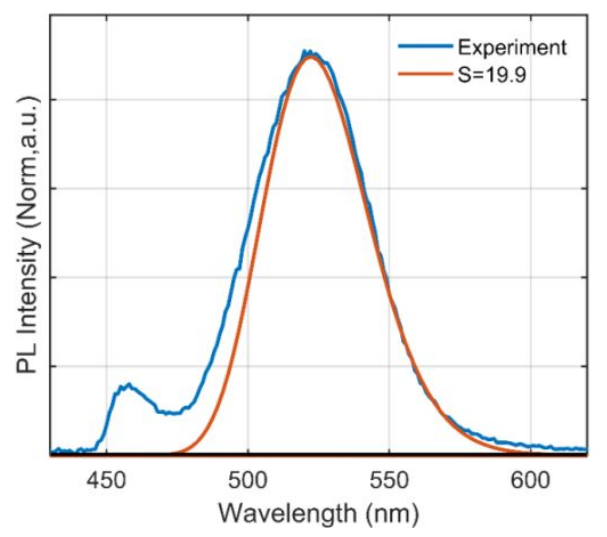

Figure S20 Calculated STS emission line shape with $S=19.9$. Normalized experimental PL line shape and calculated STS emission line shape, respectively. 
Table S1 Gaussian fitting results of PL spectrum of CdSe NPL SLs

\begin{tabular}{llllll}
\hline A1 & A2 & FWHM1 (meV) & FWHM2 (meV) & P1 (nm) & P2 (nm) \\
\hline 79160 & 1360000 & 74.5 & 227.8 & 456.4 & 516.4 \\
\hline
\end{tabular}

Table S2 Analytic peak information of XPS data

\begin{tabular}{|c|c|c|c|c|c|c|c|c|c|c|}
\hline Element & Peak & $\begin{array}{c}\text { Peak BE } \\
(\mathrm{eV})\end{array}$ & $\begin{array}{c}\text { Height } \\
\text { (CPS) }\end{array}$ & $\begin{array}{c}\text { FWHM } \\
(\mathrm{eV})\end{array}$ & $\begin{array}{c}S_{P A} \\
\text { (CPS.eV) }\end{array}$ & $\operatorname{ECF}\left(\mathrm{KE}^{0.6}\right)$ & SF & TXFN & $\begin{array}{c}S_{N P A} \\
\text { (CPS.eV) }\end{array}$ & $\begin{array}{c}\text { Atomic } \\
(\%)\end{array}$ \\
\hline \multirow{2}{*}{$\mathrm{Cd}$} & $\mathrm{Cd} \mathrm{3d}_{5 / 2}$ & 404.9 & 69229.8 & 1.22 & 81994 & 66.1 & 29 & 5338.87 & \multirow{2}{*}{0.0136} & \multirow{2}{*}{48.7} \\
\hline & $\mathrm{Cd} 3 \mathrm{~d}_{3 / 2}$ & 411.7 & 53220.1 & 1.21 & 56907 & 65.9 & 29 & 5338.87 & & \\
\hline $\mathrm{Se}$ & Se 3d & 53.9 & 6020.1 & 1.68 & 9307 & 78.3 & 1.6 & 5189.64 & 0.0143 & 51.3 \\
\hline
\end{tabular}

Postscript: $S_{P A}$ : peak area; ECF: energy compensation factor; SF: sensitivity factor; TXFN: transmission function of each element; $S_{N P A}$ normalized peak area

Table S3 $g$-factors for electron and hole in $\mathrm{CdX}(\mathrm{X}=\mathrm{S}, \mathrm{Se}, \mathrm{Te})$ nanocrystals

\begin{tabular}{ccc}
\hline $\begin{array}{c}\text { Carrier } \\
\text { Type }\end{array}$ & g-factor & Reference \\
\hline & 1.84 & {$[9]$} \\
Electron & 1.74 & {$[10]$} \\
& 1.68 & {$[11]$} \\
\hline & 2.002 & {$[12]$} \\
& 2.003 & {$[13]$} \\
Hole & 2.003 & {$[14]$} \\
& 2.004 & {$[15]$} \\
\hline
\end{tabular}


Table S4 Fitting results for the recovery dynamics in TA measurements

\begin{tabular}{|c|c|c|c|c|c|}
\hline Sample & State & $\tau_{1}(\mathrm{ps})$ & $\tau_{2}(p s)$ & $\tau_{3}(\mathrm{~ns})$ & Average (ns) \\
\hline Monodispersed & Exciton & $0.62 \pm 0.02(1 \%)$ & $\begin{array}{c}18.3 \pm 0.1 \\
(10 \%)\end{array}$ & $\begin{array}{c}0.42 \pm 0.01 \\
(89 \%)\end{array}$ & 0.36 \\
\hline \multirow{2}{*}{ SL } & Exciton & $1.9 \pm 0.1(2 \%)$ & $28.6 \pm 0.3(8 \%)$ & $\begin{array}{c}0.41 \pm 0.1 \\
(89 \%)\end{array}$ & 0.36 \\
\hline & STS & $4.3 \pm 0.3(52.0 \%)$ & $\begin{array}{l}41.5 \pm 0.8 \\
(26.3 \%)\end{array}$ & $\begin{array}{c}14.3 \pm 1.2 \\
(21.7 \%)\end{array}$ & 3.0 \\
\hline
\end{tabular}

Table S5 Multi-Lorentz peak fitting of Figure S18

\begin{tabular}{lllll}
\hline Zone 1 & & Position $\left(\mathbf{c m}^{-1}\right)$ & Width $\left(\mathbf{c m}^{-1}\right)$ & Weight \\
\hline \multirow{2}{*}{ Monodispersed } & Peak 1 & $205.9 \pm 0.1$ & $9.35521 \pm 0.3$ & $94 \%$ \\
& Peak 2 & $198.6 \pm 0.4$ & $4.10877 \pm 1.3$ & $6 \%$ \\
\hline \multirow{2}{*}{ SL } & Peak 1 & $208.2 \pm 0.6$ & $12.8 \pm 1.3$ & $49 \%$ \\
& Peak 2 & $199.1 \pm 1.2$ & $11.3 \pm 2.0$ & $51 \%$ \\
\hline
\end{tabular}

Table S6 Multi-Lorentz peak fitting of Figure 3d in the main text

\begin{tabular}{|c|c|c|c|c|}
\hline Zone 2 & & Position $\left(\mathrm{cm}^{-1}\right)$ & Width $\left(\mathrm{cm}^{-1}\right)$ & Weight (\%) \\
\hline \multirow{8}{*}{ SL } & Peak 1 & $23.7 \pm 0.3$ & $4.3 \pm 0.7$ & 4.1 \\
\hline & Peak 2 & $41.0 \pm 1.2$ & $11.2 \pm 2.4$ & 27.8 \\
\hline & Peak 3 & $48.2 \pm 0.6$ & $6.4 \pm 2.5$ & 14.3 \\
\hline & Peak 4 & $58.9 \pm 0.1$ & $3.2 \pm 0.4$ & 15.5 \\
\hline & Peak 5 & $78.5 \pm 0.1$ & $5.0 \pm 0.5$ & 27.0 \\
\hline & Peak 6 & $98.4 \pm 0.1$ & $1.3 \pm 0.4$ & 0.6 \\
\hline & Peak 7 & $106.6 \pm 0.8$ & $6.1 \pm 1.1$ & 5.9 \\
\hline & Peak 8 & $117.4 \pm 0.3$ & $4.3 \pm 0.8$ & 4.9 \\
\hline
\end{tabular}




\section{References:}

1. Widulle, F.; Kramp, S.; Pyka, N. M.; Göbel, A.; Ruf, T.; Debernardi, A.; Lauck, R.; Cardona, M., The phonon dispersion of wurtzite CdSe. Physica B: Condensed Matter. 1999, 263264, 448-451.

2. $\quad$ Stadler, W.; Hofmann, D. M.; Alt, H. C.; Muschik, T.; Meyer, B. K.; Weigel, E.; MullerVogt, G.; Salk, M.; Rupp, E.; Benz, K. W., Optical investigations of defects in Cd1-xZnxTe. Physical Review B. 1995, 51 (16), 10619-10630.

3. Toyozawa, Y., Theory of line-shapes of the exciton absorption bands. Prog. Theor. Phys. 1958, 20 (1), 53-81.

4. Christodoulou, S.; Climente, J. I.; Planelles, J.; Brescia, R.; Prato, M.; Martin-Garcia, B.; Khan, A. H.; Moreels, I., Chloride-Induced Thickness Control in CdSe Nanoplatelets. Nano Lett. 2018, 18 (10), 6248-6254.

5. de Jong, M.; Seijo, L.; Meijerink, A.; Rabouw, F. T., Resolving the ambiguity in the relation between Stokes shift and Huang-Rhys parameter. PCCP. 2015, 17 (26), 16959-16969.

6. Wang, X.; Meng, W.; Liao, W.; Wang, J.; Xiong, R. G.; Yan, Y., Atomistic Mechanism of Broadband Emission in Metal Halide Perovskites. J Phys Chem Lett. 2019, 10 (3), 501-506.

7. Huang, K.; Rhys, A., Theory of light absorption and non-radiative transitions in F-centres. Proceedings of the Royal Society of London. Series A. Mathematical and Physical Sciences. 1950, 204 (1078), 406-423.

8. $\quad$ Son, J. S.; Wen, X. D.; Joo, J.; Chae, J.; Baek, S. I.; Park, K.; Kim, J. H.; An, K.; Yu, J. H.; Kwon, S. G.; Choi, S. H.; Wang, Z.; Kim, Y. W.; Kuk, Y.; Hoffmann, R.; Hyeon, T., Large-scale soft colloidal template synthesis of $1.4 \mathrm{~nm}$ thick CdSe nanosheets. Angew. Chem. Int. Ed. Engl. 2009, 48 (37), 6861-6864.

9. $\quad$ van Schooten, K. J.; Huang, J.; Baker, W. J.; Talapin, D. V.; Boehme, C.; Lupton, J. M., Spin-Dependent Exciton Quenching and Spin Coherence in CdSe/CdS Nanocrystals. Nano Lett. 2013, 13 (1), 65-71.

10. Kudlacik, D.; Sapega, V. F.; Yakovlev, D. R.; Kalitukha, I. V.; Shornikova, E. V.; Rodina, A. V.; Ivchenko, E. L.; Dimitriev, G. S.; Nasilowski, M.; Dubertret, B., Single and double electron spin-flip Raman scattering in CdSe colloidal nanoplatelets. Nano Lett. 2019, 20 (1), 517-525.

11. Shornikova, E. V.; Biadala, L.; Yakovlev, D. R.; Feng, D.; Sapega, V. F.; Flipo, N.; Golovatenko, A. A.; Semina, M. A.; Rodina, A. V.; Mitioglu, A. A., Electron and hole g-factors and spin dynamics of negatively charged excitons in $\mathrm{CdSe} / \mathrm{CdS}$ colloidal nanoplatelets with thick shells. Nano Lett. 2018, 18 (1), 373-380.

12. Taylor, A.; Filipovich, G.; Lindeberg, G., Identification of Cd vacancies in neutronirradiated CdS by electron paramagnetic resonance. Solid State Commun. 1971, 9 (13), 945-947.

13. Keeble, D. J.; Thomsen, E. A.; Stavrinadis, A.; Samuel, I. D.; Smith, J. M.; Watt, A. A., Paramagnetic point defects and charge carriers in $\mathrm{PbS}$ and $\mathrm{CdS}$ nanocrystal polymer composites. The Journal of Physical Chemistry C. 2009, 113 (40), 17306-17312.

14. Kurita, Y.; Gordy, W., Electron Spin Resonance in a Gamma-Irradiated Single Crystal of L-Cystine Dihydrochloride. The Journal of Chemical Physics. 1961, 34 (1), 282-288.

15. Almeida, A. n. J.; Sahu, A.; Riedinger, A.; Norris, D. J.; Brandt, M. S.; Stutzmann, M.; Pereira, R. N., Charge trapping defects in CdSe nanocrystal quantum dots. The Journal of Physical Chemistry C. 2016, 120 (25), 13763-13770. 